Educación Física y Ciencia, vol. 21, n 2, e077, abril-junio 2019. ISSN 2314-2561

Universidad Nacional de La Plata.

Facultad de Humanidades y Ciencias de la Educación.

Departamento de Educación Física

\title{
Locus Percibido de Causalidad en adolescentes mexicanos. Comparaciones por género
}

\author{
Perceived Locus of Causality in Mexican adolescents. Gender Comparisons
}

\author{
Martha Ornelas Contreras \\ Facultad de Ciencias de la Cultura Física, DES Salud, Universidad Autónoma de Chibuahua, \\ México
}

Luis Humberto Ornelas Contreras

OPD Hospital Civil de Guadalajara, Universidad de Guadalajara,

México

Miguel Conchas Ramirez

Facultad de Ciencias de la Cultura Física, DES Salud, Universidad Autónoma de Chibuahua,

México

Yunuen Socorro Rangel Ledezma

Facultad de Ciencias de la Cultura Física, DES Salud, Universidad Autónoma de Chibuahua, México

Judith Margarita Rodriguez Villalobos

Facultad de Ciencias de la Cultura Física, DES Salud, Universidad Autónoma de Chibuahua, México jurodrig@uach.mx

\section{RESUMEN:}

El objetivo de esta investigación consistió en comparar los perfiles de motivación hacia las clases de educación física de alumnos y alumnas de secundaria mexicanos. .La muestra total fue de 549 sujetos; 256 mujeres y 293 hombres, con una edad media de 12,9 años $(\mathrm{DE}=0,9)$ y 12,8 años $(\mathrm{DE}=1,0)$ respectivamente. El abordaje adoptado en la investigación se enmarcó dentro de un enfoque cuantitativo con un diseño descriptivo tipo encuesta. En los resultados del análisis multivariante de la varianza, seguido por los análisis de varianza univariados se encontró que, mostraron que los hombres reportan mayores niveles de motivación intrínseca ( $\mathrm{F}$ $=5,27, \mathrm{p}<, 05)$, regulación integrada $(\mathrm{F}=6,74, \mathrm{p}<, 01)$, regulación identificada $(\mathrm{F}=3,96, \mathrm{p}<, 05)$ y regulación introyectada $(\mathrm{F}$ $=12,28, \mathrm{p}<, 001)$ que las mujeres y sin diferencias significativas con respecto a regulación externa y desmotivación, de tal forma que las mujeres al manifestar niveles más bajos de motivación presentan un mayor riesgo de abandonar la práctica de actividad física que los hombres.

Palabras Clave: Motivación, Clase de educación física, Autoregulación, Locus.

\section{Abstract:}

The aim of this research was to compare the profiles of motivation towards Physical Education classes of Mexican high school students. The total sample was 549 subjects; 256 women and 293 men, with an average age of 12.9 years $(S D=0.9)$ and 12.8 years $(S D=1.0)$ respectively. The study was a quantitative approach with a survey descriptive design. In the results of the multivariate analysis of variance, followed by the univariate analysis of variance, it was found that men report higher levels of intrinsic motivation $(F=5.27, p<0.05)$, integrated regulation $(F=6) ., 74, p<, 01)$, identified regulation $(F=3.96, p<, 05)$ and introjected regulation $(\mathrm{F}=12.28, \mathrm{p}<, 001)$ than women and without significant differences with respect to external regulation and demotivation. Therefore, as manifesting lower levels of motivation, women have a higher risk of abandoning physical activity than men.

KEYWORDS: Motivation, Physical education class, Self-regulation, Locus. 


\section{INTRODUCCIÓN}

Las conductas sedentarias generan influencia sobre el estado de salud de los adolescentes (Lavielle, Pineda, Jáuregui \& Castillo, 2014). En esta edad la educación física (EF) se constituye el mecanismo que favorece la práctica de la actividad física (AF) (Moreno, Parra \& González-Cutre, 2008) en la expectativa de que los patrones aprendidos en esta etapa, permanezcan en la edad adulta. Este no es necesariamente un fenómeno observado en la realidad debido a que existen elevados índices de sedentarismo en la edad adulta (Gondim \& Lessa, 2005) de manera que la persona se convierte en sujeto expuesto a otro tipo de enfermedad como la depresión (Wilson-Escalante, Sánchez-Rodríguez \& Mendoza-Núñez, 2009).

Valorar los parámetros asociados a la práctica de la EF resulta fundamental en diseño de estrategias orientadas a identificar la adherencia y permanencia en programas de AF dentro y fuera del centro escolar (González-Cutre, Sicilia \& Moreno, 2011). En este caso, La Escala de Locus Percibido de Causalidad (PLOC) se constituye un instrumento válido que ofrece la posibilidad de detectar la motivación del alumnado hacía las clases de EF (Ferriz, González-Cutre \& Sicilia, 2015; Goudas, Biddle \& Fox, 1994). La motivación puede ser identificada dentro de la teoría de la autodeterminación (TAD) cuyas normas se rigen por categorizar la motivación dentro de cinco ejes: 1) motivación intrínseca, 2) motivación extrínseca, 3) regulación externa, 4) regulación introyectada y 5) desmotivación (Ferriz et al., 2015); bajo este precepto se pueden identificar las causas por las cuales un individuo prefiere o le atribuye un comportamiento hacia un fenómeno, en este caso la preferencia o no hacia la práctica de EF.

La motivación es un fenómeno psicológico multifactorial que difícilmente se puede encuadrar bajo un solo instrumento de medición, por este motivo la valoración mediante el TAD pudiera ser razón de controversia dado que se genera a partir de un autoreporte y por lo tanto de las desventajas que los acompañan (McDonald, 2008). Sin embargo estudios como el Moreno, González-Cutre \& Chillón (2009) señalan que el Locus ha sido examinado mediante procesamientos estadísticos como análisis factoriales confirmatorios, análisis de la invarianza factorial, análisis de correlaciones entre factores, fiabilidad a través del alfa de Cronbach, estabilidad temporal test-retest y validez de criterio en los cuales se reportaron resultados por encima de 0,70 en el cual se concluye valores aceptables en la adaptación de la prueba del idioma nativo al castellano (Moreno et al, 2009).

Por otro lado, se han reportado dificultades en la medición del Locus en estudiantes de relación a las propiedades interculturales, ya que en estudiantes de ascendencia oriental se detectaron deficiencias en la consistencia interna. Cabe mencionar que el estudio se realizó en el Reino Unido y que en los estudiantes nativos no se presentaron inconsistencias (Lonsdale, Sabiston, Taylor \& Ntoumanis, 2011) por lo que se requieren realizar adaptaciones al idioma, en cuyo caso el cuestionario PLOC ha sido adaptado al castellano considerando los elementos estadísticos pertinentes (Ferriz et al., 2015; Goudas et al., 1994; Moreno et al., 2009).

La literatura que se ha analizado aborda el tema como parte de las estrategias necesarias para mejorar y potencializar las clases de educación física, encontrando evidencias de asociación de las clases con la motivación intrínseca de realizar actividades físicas extraescolares, lo cual es uno de los principales propósitos que se intentan atender en los programas educativos de la asignatura (Gutierrez, 2017). La motivación puede llegar a ser compleja por la variedad de contextos culturales propios de cada región, sin embargo en estudios como el de Franco, Coterón, Gómez, Brito, \& Martínez (2017) se observaron patrones motivacionales similares entre jóvenes de cuatro países: España, Argentina, Ecuador y Colombia, enfatizando la importancia de este tipo de estudios para predecir la intención futura de ser físicamente activo.

El género parece ser una de las propiedades más estudiadas con respecto a la motivación de practicar AF. Hasta el momento, los resultados generalmente señalan que existe mayor intención de realizar AF en estudiantes varones (Lim \& Wang, 2009; Cabello, Moyano, \& Tabernero, 2018) o que el estilo de vida saludable y de práctica de AF es más frecuentemente observada en hombres (Granero-Gallegos, Baena- 
Extremera, Sánchez-Fuentes \& Martínez-Molina, 2014; Sallis, Zakarian, Hovell \& Hofstetter, 1996; Trost et al., 2002) en el caso de las mujeres, prefieren actividades sociales o de cuidado personal (Murcia, Galindo \& Villodre, 2006; Azofeifa, 2018). Sin embargo, algunos estudios recientes sugieren resultados diferentes (Baños, Ortiz-Camacho, Baena-Extremera, \& Zamarripa, 2018) que mantienen controversia en el análisis de la motivación hacia la práctica de actividad física.

En este estudio se intentó determinar las diferencias y similitudes entre adolescentes hombres y mujeres mexicanos en cuanto a sus perfiles de motivación hacia las clases de educación física. En consecuencia, esta investigación pretendió, como investigación aplicada, aportar información que se traduzca en una práctica educativa de mayor calidad en el contexto de atención a la diversidad; contribuyendo al saber pedagógico en el esclarecimiento de los factores que conforman un modelo de desarrollo humano integral; bajo la premisa de que los esfuerzos educativos deben enfocarse hacia el aumento de los sentimientos de autovaloración y competencia de los estudiantes, fortaleciendo la autoestima y el autoconcepto, lo que a su vez favorecerá la motivación hacia el logro, las relaciones interpersonales y en general la forma particular de desenvolverse frente a diversas tareas y desafíos que se les presenten.

\section{Metodología}

\section{Participantes}

Participaron en el estudio 549 todos alumnos y alumnas de secundaria de la Ciudad de Chihuahua México, 256 mujeres y 293 hombres; con una edad media de 12,9 años $(\mathrm{DE}=0,9)$ y 12,8 años $(\mathrm{DE}=1.0)$ respectivamente.

La muestra se obtuvo mediante un muestreo por conveniencia, tratando de abarcar la representatividad de los tres grado de secundaria que se ofrecen en las escuelas de la Ciudad de Chihuahua, México.

\section{Instrumento}

Para medir los perfiles de motivación hacia las clases de educación física se utilizó la Escala de Locus Percibido de Causalidad (PLOC-2) adaptada por Ferriz et al. (2015) la cual consta de 6 factores con 4 ítems cada uno, que miden la motivación intrínseca, la regulación integrada, la regulación identificada, la regulación introyectada, la regulación externa y la desmotivación. Para nuestro estudio se hicieron tres adaptaciones a la versión de Ferriz y colaboradores: (a) en la versión original se puntúa con siete opciones de respuesta 1 (totalmente en desacuerdo) a 7 (totalmente de acuerdo); en la versión utilizada en la presente investigación el sujeto elige entre once posibles respuestas 0 (totalmente en desacuerdo) a 10 (totalmente de acuerdo); (b) la primera consistió en cambiar algunos términos utilizados en los ítems de la versión original con el fin de emplear un lenguaje más adecuado al contexto de la cultura mexicana; (c) la segunda consistió en aplicar el instrumento por medio de una computadora, permitiendo así el almacenamiento de los datos sin previa codificación, con mayor precisión y evitando errores.

\section{Diseño}

En cuanto al diseño del estudio, se utilizó un enfoque cuantitativo con un diseño descriptivo y transversal tipo encuesta (Hernández, Fernández \& Baptista, 2014). La variable independiente fue el género y las variables dependientes el promedio de las puntuaciones en los factores motivación intrínseca, regulación integrada, regulación identificada, regulación introyectada, regulación externa y desmotivación del cuestionario Locus Percibido de Causalidad (PLOC-2). 


\section{Procedimiento}

Una vez conseguido el permiso tanto de las autoridades educativas como el de las familias, se invitó a participar en el estudio a los alumnos y alumnas de secundaria de la ciudad de Chihuahua.

Los que aceptaron participar firmaron el consentimiento informado. Luego se aplicó el instrumento por medio de una computadora personal, en una sesión de aproximadamente 30 minutos; en las aulas de los centros educativos participantes.

$\mathrm{Al}$ inicio de cada sesión se hizo una pequeña introducción sobre la importancia de la investigación y de cómo acceder al instrumento. Se les solicitó la máxima sinceridad y se les garantizó la confidencialidad de los datos que se obtuvieran. Las instrucciones de cómo responder se encontraban en las primeras pantallas; antes del primer reactivo del instrumento. Al término de la sesión se les agradeció su participación.

Una vez aplicado el instrumento se procedió a recopilar los resultados por medio del módulo generador de resultados del editor de escalas versión 2.0 (Blanco et al., 2013).

\section{Análisis de datos}

Se realizaron análisis estadísticos descriptivos (medias y desviaciones estándar) para todas las variables. Posteriormente, después de verificar que los datos cumplían con los supuestos de los análisis estadísticos paramétricos, se llevó a cabo un análisis multivariante de varianza (MANOVA), seguido por los análisis de varianza univariados (ANOVAs), para examinar las diferencias entre hombres y mujeres en cuanto a su motivación hacia la clase de educación física. Por otra parte, el tamaño del efecto se estimó mediante el eta cuadrado $(\eta 2)$. Todos los análisis estadísticos se realizaron con el programa SPSS versión 21,0 para Windows. El nivel de significación estadística se estableció en $\mathrm{p}=, 05$.

\section{Resultados}

La Tabla 1 muestra los valores de las medias y las desviaciones estándar de las puntuaciones de los seis factores estudiados sobre la motivación hacia la clase de educación física, así como los resultados del MANOVA y los subsiguientes ANOVAs. Los resultados del MANOVA mostraron diferencias globales estadísticamente significativas entre mujeres y hombres en las puntuaciones promedio de los factores de la Escala de Locus Percibido de Causalidad (PLOC-2) (Wilks' $\lambda=, 97 ; \mathrm{p}<, 01 ; \eta 2=, 03$ ). Posteriormente, los resultados de los ANOVAs mostraron que los hombres reportan mayores niveles de motivación intrínseca $(\mathrm{F}=5,27, \mathrm{p}<, 05)$, regulación integrada $(\mathrm{F}=6,74, \mathrm{p}<, 01)$, regulación identificada $(\mathrm{F}=3,96, \mathrm{p}<, 05) \mathrm{y}$ regulación introyectada $(\mathrm{F}=12,28, \mathrm{p}<, 001)$ que las mujeres y sin diferencias significativas con respecto a regulación externa y desmotivación. 
Tabla 1. Resultados MANOVA y ANOVAs para las diferencias en motivación hacia la clase de educación física de acuerdo al género

\begin{tabular}{lccccc}
\hline & $\begin{array}{c}\text { mujeres } \\
(n=256)\end{array}$ & $\begin{array}{c}\text { hombres } \\
(n=293)\end{array}$ & $F$ & $p$ & $\eta^{2}$ \\
\hline Motivación intrínseca & $7,51(2,22)$ & $7,94(2,09)$ & 5,27 & $<, 05$ &, 01 \\
Regulaciónintegrada & $7,22(2,30)$ & $7,72(2,17)$ & 6,74 & $<, 01$ &, 01 \\
Regulaciónidentificada & $7,78(2,11)$ & $8,14(2,03)$ & 3,96 & $<, 05$ &, 01 \\
Regulación introyectada & $4,45(1,66)$ & $3,96(1,60)$ & 12,28 & $<, 001$ &, 02 \\
Regulación extema & $4,67(2,68)$ & $4,44(2,58)$ & 1,01 & $>, 05$ &, 00 \\
Desmotivación & $6,31(2,75)$ & $5,89(2,87)$ & 3,02 & $>, 05$ &, 00 \\
\hline
\end{tabular}

Nota. Los valores descriptivos se presentan como media (desviación estándar)

\section{Discusión}

El objetivo de este estudio fue determinar las diferencias y similitudes entre adolescentes mexicanos en cuanto a sus perfiles de motivación hacia la clase de educación física, aun cuando no se encontraron diferencias significativas entre las adolescentes y los adolescentes en cuanto a su regulación externa y desmotivación, las mujeres muestran una menor motivación hacia la clase de educación física en el resto de las dimensiones, incluida la regulación introyectada donde el tamaño del efecto es el más importante resultados que en general concuerdan con los obtenidos por Granero-Gallegos et al., 2014; Trigueros, Sicilia, Alcaraz-Ibáñez \& Dumitru, 2017; Cabello, 2018; de tal forma que las mujeres al manifestar niveles más bajos de motivación presentan un mayor riesgo de abandonar la práctica de actividad física que los hombres, lo que conlleva a problemas de salud a futuro, como obesidad en la edad adulta, cardiopatías o patologías asociadas a el sedentarismo.

\section{Conclusiones}

De los resultados mostrados, de su análisis y de su discusión, se pueden obtener las siguientes conclusiones:

1. Los adolescentes hombres en relación a las adolescentes mujeres, manifiestas una mayor motivación hacia la clase de educación física, favoreciendo la práctica de actividad física en mayor medida que la adolescentes mujeres.

2. Las diferencias encontradas entre las poblaciones estudiadas en cuanto a su motivación hacia la clase de educación física, sugieren además que al diseñar cualquier tipo de intervención que tenga como objetivo el aumento de dicha motivación, habrá que tomar en cuenta a la variable género; no obstante, es preciso desarrollar más investigación al respecto pues el tema trasciende del todo los alcances de la presente investigación.

Por último, cuando menos dos limitaciones están presentes en este trabajo. La primera es que los participantes son solo estudiantes de secundaria mexicanos, lo que supone una amenaza para la posibilidad de generalizar estos resultados. Ampliar la muestra (agregando por ejemplo jóvenes) es un área de trabajo de cara 
al futuro. La segunda limitación proviene del propio instrumento de medición, que se basa en el autoinforme y que por ello puede contener los sesgos que se derivan de la deseabilidad social.

\section{Agradecimientos}

Este estudio es parte de un proyecto financiado por la Secretaría de Educación Pública-Subsecretaría de Educación Superior-Dirección de Superación Académica- Programa para el Desarrollo Profesional Docente (DE-13 -6894).

\section{REFERENCIAS}

Azofeifa, C. A. (2018). Comparación entre los motivos para la práctica de la actividad física según el género y el tiempo de práctica en un grupo de estudiantes. MH Salud, 14(2), 1-14.

Baños, R., Ortiz-Camacho, M. d. M., Baena-Extremera, A., \& Zamarripa, J. (2018). Efecto del género del docente en la importancia de la Educación Física, clima motivacional, comportamientos disruptivos, la intención de práctica futura y rendimiento académico. Retos, 33, 252-257.

Blanco, H. , Ornelas, M. , Tristán, J. L. , Cocca, A. , Mayorga-Vega, D. , López-Walle, J. \& Viciana, J. (2013). Editor for creating and applying computerise surveys. Procedia Social and Behavioral Sciences, 106, 935-940. doi: htt ps://doi.org/10.1016/j.sbspro.2013.12.105

Cabello, A., Moyano, M., \& Tabernero, C. (2018). Procesos psicosociales en Educación Física: actitudes, estrategias y clima motivacional percibido. Retos, 34, 19-24.

Ferriz, R. , González-Cutre, D. \& Sicilia, Á. (2015). Revisión de la Escala del Locus Percibido de Causalidad (PLOC) para la inclusión de la medida de la regulación integrada en educación física. Revista de psicología del deporte, 24(2), 329-338.

Franco, E., Coterón, J., Gómez, V., Brito, J., \& Martínez, H. (2017). Influencia de la motivación y del flow disposicional sobre la intención de realizar actividad físico-deportiva en adolescentes de cuatro países. Retos, 31, 46-51.

Gondim, F. J. \& Lessa, I. (2005). Prevalência e fatores associados ao sedentarismo no lazer em adultos Prevalence and variables associated with leisure-time sedentary lifestyle in adults. Cadernos de Saúde Pública, 21(3), 870-877. doi: https://doi.org/10.1590/S0102-311X2005000300021

González-Cutre, D., Sicilia, Á. \& Moreno, J. A. (2011). Un estudio cuasi-experimental de los efectos del clima motivador tarea en las clases de Educación Física. Revista de Educación (356), 677-700. doi: https://doi.org/1 0-4438/1988-592X-RE-2010-356-056

Goudas, M., Biddle, S. \& Fox, K. (1994). Perceived locus of causality, goal orientations, and perceived competence in school physical education classes. British Journal of Educational Psychology, 64(3), 453-463.

Granero-Gallegos, A., Baena-Extremera, A., Sánchez-Fuentes, J. A. \& Martínez-Molina, M. (2014). Perfiles motivacionales de apoyo a la autonomía, autodeterminación, satisfacción, importancia de la educación física e intención de práctica física en tiempo libre. Cuadernos de Psicología del Deporte, 14(2), 59-70.

Gutierrez, M. (2017). Efecto de las actitudes hacia la Educación Física sobre los motivos de práctica deportiva fuera del horario escolar. Sportis. Scientific Technical Journal, 3(1), 123-140.

Hernández, R. , Fernández, C. \& Baptista, P. (2014). Metodología de la investigación. México: McGraw- Hill.

Lavielle, P. , Pineda, V. , Jáuregui, O. \& Castillo, M. (2014). Actividad física y sedentarismo: Determinantes sociodemográficos, familiares y su impacto en la salud del adolescente. Revista de salud pública, 16(2), 161-172.

Lim, B. S. C. \& Wang, C. K. J. (2009). Perceived autonomy support, behavioural regulations in physical education and physical activity intention. Psychology of Sport and Exercise, 10(1), 52-60. doi: https://doi.org/10.1016/ j.psychsport.2008.06.003

Lonsdale, C., Sabiston, C. M., Taylor, I. M. \& Ntoumanis, N. (2011). Measuring student motivation for physical education: examining the psychometric properties of the perceived locus of causality questionnaire and the 
situational motivation scale. Psychology of Sport and Exercise, 12(3), 284-292. doi: https://doi.org/10.1016/j .psychsport.2010.11.003

McDonald, J. D. (2008). Measuring personality constructs: The advantages and disadvantages of self-reports, informant reports and behavioural assessments. Enquire, 1(1), 75-94.

Moreno, J. A. , González-Cutre, D. \& Chillón, M. (2009). Preliminary validation in Spanish of a scale designed to measure motivation in physical education classes: the Perceived Locus of Causality (PLOC) Scale. The Spanish Journal of Psychology, 12(1), 327-337.

Moreno, J. A. , Parra, N. \& González-Cutre, D. (2008). Influencia del apoyo a la autonomía, las metas sociales y la relación con los demás sobre la desmotivación en educación física. Psicothema, 20(4), 636-641.

Murcia, J. A., Galindo, M. C. \& Villodre, N. A. (2006). Actitudes hacia la práctica físico-deportiva según el sexo del practicante. Revista Internacional de Ciencias del Deporte, 2(3), 20-43. doi: https://doi.org/10.5232/ricyde2 006.00302

Sallis, J. F., Zakarian, J. M., Hovell, M. F. \& Hofstetter, C. R. (1996). Ethnic, socioeconomic, and sex differences in physical activity among adolescents. Journal of clinical epidemiology, 49(2), 125-134.

Trigueros, R., Sicilia, Á., Alcaraz-Ibáñez, M. \& Dumitru, D. C. (2017). Adaptación y validación española de la escala revisada del locus percibido de causalidad (PLOC-R) en educación física. Cuadernos de Psicología del Deporte, $17(1), 25-32$.

Trost, S. G., Pate, R. R., Sallis, J. F., Freedson, P. S., Taylor, W. C., Dowda, M. \& Sirard, J. (2002). Age and gender differences in objectively measured physical activity in youth. Medicine and science in sports and exercise, 34(2), 350-355.

Wilson-Escalante, L. K. , Sánchez-Rodríguez, M. A. \& Mendoza-Núñez, V. M. (2009). Sedentarismo como factor de riesgo de trastornos depresivos en adultos mayores. Un estudio exploratorio. Revista de la Facultad de Medicina UNAM, 52(6), 244-247. 